\title{
Dosimetric feasibility on hypofractionated intensity-modulated radiotherapy and simultaneous integrated boost for locally advanced unresectable pancreatic cancer with helical tomotherapy
}

\author{
Feng Teng', Lingling Meng', Fuhai Zhu' ${ }^{3}$, Gang Ren ${ }^{3}$ \\ ${ }^{1}$ Department of Radiation Oncology, China-Japan Friendship Hospital, Beijing, China; ${ }^{2}$ Department of Radiotherapy, First Medical Center of \\ Chinese PLA General Hospital, Beijing, China; ${ }^{3}$ Department of Radiotherapy, Air Force Medical Center of PLA, Beijing, China \\ Contributions: (I) Conception and design: G Ren; (II) Administrative support: G Ren; (III) Provision of study materials or patients: F Zhu; (IV) \\ Collection and assembly of data: F Teng, L Meng; (V) Data analysis and interpretation: F Teng, L Meng; (VI) Manuscript writing: All authors; (VII) \\ Final approval of manuscript: All authors. \\ Correspondence to: Gang Ren, MD. Department of Radiotherapy, Air Force Medical Center of PLA, 30 Fucheng Road, Haidian District, Beijing \\ 100142, China. Email: rgsn122@163.com.
}

Background: This dosimetric study on locally advanced pancreatic cancer (LAPC) and the surrounding gastrointestinal organs at risk (OARs) aimed at exploring the potential of further improving the internal dose and reducing the fractionation number by concurrent hypofractionated simultaneous integrated boost (SIB) radiotherapy using helical tomotherapy (HT).

Methods: We collected computed tomography positioning images from a LAPC study of 17 consecutive patients. Gross tumor volume (GTV)1, GTV2, and GTV3 were defined as the GTV minus a margin of 3,6 , and $9 \mathrm{~mm}$ from the external part in all directions, respectively. Under the same physical parameters and limited dose on normal organs, each case had 4 sets of SIB radiotherapy plans. Upon dose escalation, we statistically analyzed the difference of dosimetric parameters received by the OARs between group A [planning target volume (PTV)/GTV=50 Gy/70 Gy] and the other groups. According to the equivalent bioradiotherapy formula, we calculated the hypofractionated standard dose by converting the average tolerated dose of each OAR with the corresponding number of fractions. Then, we compared the dose and volume parameters of the gastrointestinal tract from the less-than-20-fraction modes with the corresponding gastrointestinal hypofractionated standard dose.

Results: For dose escalation, although there were a few differences in the parameters of the OAR between group A and group D, all OAR doses of group D (PTV/GTV/GTV1/GTV2/GTV3= $50 \mathrm{~Gy} / 70 \mathrm{~Gy} / 80 \mathrm{~Gy} / 90 \mathrm{~Gy} / 100 \mathrm{~Gy}$ ) were within the limited dose range. In the hypofractionated mode, there was a statistically significant difference between the gastrointestinal dose-volume parameters and the doselimiting reference standard when the fraction number was less than 14 or 15 for group A or D, respectively.

Conclusions: The dose of the internal target can be increased to 100 Gy with 15 fractions in the hypofractionated SIB radiotherapy for LAPC with HT. The corresponding tolerance dose of OARs may also be acceptable.

Keywords: Locally advanced pancreatic cancer (LAPC); hypofractionated radiotherapy; simultaneous integrated boost (SIB); dosimetry; helical tomotherapy (HT)

Submitted Mar 10, 2021. Accepted for publication Apr 20, 2021.

doi: 10.21037/jgo-21-160

View this article at: http://dx.doi.org/10.21037/jgo-21-160 


\section{Introduction}

Pancreatic cancer is a severe malignancy, and its 5-year survival rate is as low as $9 \%$ (1). Most pancreatic cancers are locally advanced and unresectable, exhibiting a poor prognosis after they are first diagnosed. Currently, radiotherapy combined with chemotherapy has become the standard treatment option for locally advanced pancreatic cancer (LAPC) (2). The recommended total dose of radiotherapy for pancreatic cancer is 50 and $40 \mathrm{~Gy}$ under the conventional radiotherapy and SBRT model, respectively. Furthermore, it is generally believed that a biologically effective dose (BED) beyond 100 Gy can potentially achieve radical cure (3). However, conventional intensity-modulated radiotherapy (IMRT) or SBRT can hardly reach that target due to the need for sparing the nearby organs at risk (OARs), especially the gastrointestinal tract.

Over the past few decades, high-precision radiotherapy technologies, such as helical tomotherapy (HT), have emerged. Within the context of pancreatic cancer treatment, this has enabled the sparing of the surrounding gastrointestinal tract without compromising the coverage of radiation dose to the tumor target. Moreover, the protective effect of HT on peripancreatic normal organs further allows for the increase in the radiation dose to the tumor (4).

The ability of conventional radiotherapy to deliver a high dose in pancreatic carcinoma is limited, due to the tumor's close proximity to the gastrointestinal tract. Radiotherapy (60 Gy) combined with chemotherapy was used for LAPC treatment by the Federation Francophone de Cancerologie Digestive-Societe Francaise de Radiotherapie Oncologique (FFCD-SFRO); However, the long-term survival was found to be affected by gastrointestinal (GI) toxicities (5). The conventional radiotherapy was administered at a total dose of 45 to 54 Gy and the single fraction dose of 1.8 to $2.5 \mathrm{~Gy}$, for the purpose of adjuvant therapy and palliative therapy. A phase III result suggested that conventional radiotherapy combined with gemcitabine could prolong survival compared with chemotherapy alone (6). Moreover, results from the LAP07 trial result suggested that chemoradiotherapy could slow LAPC local progression (chemoradiotherapy vs. chemotherapy $32 \%$ vs. $46 \%$, $\mathrm{P}=0.03$ ) with no increase in grade 3 to 4 gastrointestinal toxicity. However, this study did not demonstrate an overall survival benefit from radiation at standard doses (7).

With the development of radiotherapy technology, including volumetric-modulated radiation therapy (VMAT),
HT, gamma knife, image-guided radiation therapy (IGRT), 4-dimensional computed tomography (4D-CT), and breath-hold technique (BHT), it is now possible when treating pancreatic cancer to spare the gastrointestinal tract while still covering the target volume (8-13). Compared with IMRT, HT can be performed with a dosimetrically comparable conformity index (CI) and lower gastrointestinal toxicity in pancreatic cancer (14). Indeed, under the same uniformity index (UI) and CI, the exposure doses of the stomach and small intestine in the HT plan were found to be lower than those in the IMRT plan (9). The protective effect of precision radiotherapy technology on normal peripancreatic organs allows for a further increase in the radiation dose of the tumor. The radiation dose in the target area is proportional to the curative effect of radiotherapy. One study showed that patients who received a biologically effective dose (BED) of $>70$ Gy using $\alpha / \beta=10$ Gy for tumor showed a superior overall survival compared with those receiving a $\mathrm{BED}$ of $\leq 70$ Gy (17.8 vs. 15.0 months, $\mathrm{P}$ value $=0.03)(15)$. Generally speaking, a BED greater than or equal to $100 \mathrm{~Gy}$ is believed to able to achieve radical cure of the tumor. Therefore, high dose irradiation of pancreatic cancer can bring about survival benefits.

Given the poor prognosis and gastrointestinal toxicities induced by radiotherapy, intensification by dose escalation can be crucial yet challenging for the treatment of pancreatic cancer. Dose escalation in the simultaneous integrated boost (SIB) is a feasible way to avoid high doses in the surrounding gastrointestinal tract. We have successfully performed body gamma knife for pancreatic cancer for decades (2). The dosage of the gamma knife distributes in a pattern like the skin of an onion, where the dose escalates toward the internal target, and the inner tumor can therefore receive 2 times as much of the dose as the planning target volume (PTV). Increasing the fractionation dose and reducing the number of fractionations by hypofractionated radiotherapy or stereotactic body radiation therapy (SBRT) is another way to increase the total radiation dose. The high-dose fractionation mode has been widely used in solid tumors, such as lung cancer and liver cancer. At present, the commonly used high-dose fractionation mode for pancreatic cancer is $30-35$ Gy/3-5 times (16), but it is difficult to improve the tumor exposure to the BED this way. For pancreatic cancer radiotherapy, even though the hypofractionated radiotherapy is likely the most suitable radiation dose mode, there is yet no consistency in treatment time (17). A gamma knife is usually used in the treatment of pancreatic cancer for 10-17 
fractionations, and the 5-year survival rate of early-stage pancreatic cancer by gamma knife can reach 21\% (13). Recently, we successfully applied HT for the treatment of pancreatic cancer by imitating the gamma knife mode. PTV and gross tumor volume (GTV) were respectively 50 and 70 Gy with 20 fractions. The toxicity was acceptable in the phase I/II trials, but the dose-toxicity relationship has not been fully established $(18,19)$. Based on the primary dose, we conducted this dosimetric study on pancreatic cancer and OARs to explore the potential of further improving the internal dose and reducing the fractionation number by using hypofractionated SIB HT radiotherapy. This is the first study to demonstrate that applying hypofractionated SIB chemoradiotherapy using HT on the internal targets for improving the overall survival of LAPC exhibits lower gastrointestinal toxicities. We present the following article in accordance with the MDAR reporting checklist (available at http://dx.doi.org/10.21037/jgo-21-160).

\section{Methods}

\section{Patient selection}

We consecutively collected computed tomography (CT) positioning images of 17 patients with LAPC for this study. Verbal informed consent was obtained from all patients (mean age: 62, male/female $=10 / 7$ ). Due to its dosimetric design, the study did not require ethical board approval, as it did not involve any animal or human experiments or interventions. The study was conducted in accordance with the Declaration of Helsinki (as revised in 2013). During collection, the patients were in the supine position, put hands on forehead, and their bodies were fixed by thermoplastic films with a carbon fiber body-fixed frame. The fixation scope was the abdomen, and scanning range was from the liver to the top of the iliac crest edge with a $75-\mathrm{cm}$ aperture setting on CT (Siemens Emotion 16). Oral administration of $250 \mathrm{~mL}$ of $3 \%$ iodinated contrast media was performed 15 minutes before positioning. An abdominal enhanced CT scan was performed under a calm breathing state, with a thickness of $5 \mathrm{~mm}$ and reconstruction of $4 \mathrm{~mm}$ after scanning. Scanned images and data were transmitted via the network system to the doctor's workstation. The same radiotherapy physicians contoured the targets and surrounding OARs.

\section{Treatment planning}

The GTV encompassed primary pancreatic lesions and metastatic lymph nodes. The clinic tumor volume (CTV) was delineated as GTV plus a $5-\mathrm{mm}$ isotropic margin. The planning tumor volume (PTV) was defined as CTV plus a $10-\mathrm{mm}$ margin in cranial-caudal direction and a 5 -mm margin in the other directions. The margin was adjusted based on the location between the tumor and the gastrointestinal tract. GTV1, GTV2 and GTV3 were defined as the GTV minus 3, 6, and $9 \mathrm{~mm}$ in all directions, respectively. OARs include the stomach, duodenum, intestine, left and right liver, kidneys, and spinal cord.

Each patient had 4 sets of SIB radiotherapy planning. These were configured into the following groupings: group A, PTV/GTV =50 Gy/70 Gy; group B, PTV/ GTV/GTV1=50 Gy/70 Gy/80 Gy, group C, PTV/GTV/ GTV1/GTV2 =50 Gy/70 Gy/80 Gy/90 Gy; and group D, PTV/GTV/GTV1/GTV2/GTV3 =50 Gy/70 Gy/80 Gy/ 90 Gy/100 Gy.

The fractionation number of the treatment plans was carried out 20 times. The CT images with the contoured objects were transmitted to the planning system of HT (version varian Eclipse 4.0.4.17). The same physical therapist planned radiation therapy under the same physical parameters (beam width $=2.5$; pitch $=0.287$; beam intensity modulation factors 2.5 , etc.) and limited the dose to the normal organs. According to the anatomical relationship between the targets and OARs, we iteratively adjusted the objectives to generate the optimal plans. The D95\% (the prescribed dose required to include at least $95 \%$ of the target volume) and V95\% (the target volume required to receive at least $95 \%$ of the prescription dose) were used to assess plan quality. D95\% and V95\% were obtained by the dose-volume histogram (DVH) from the Treatment Planning System (TPS). The following thresholds were set for the gastrointestinal tract: D1 $\leq 55$ Gy, D $3 \leq 50 \mathrm{~Gy}$, D $5 \leq 45$ Gy. In addition, the parameters of V $20 \leq 40 \%$, V30 $\leq 30 \%$, and Dmax (maximum dose) $\leq 45$ Gy were applied to the kidney, liver, and spinal cord, respectively.

\section{Plan evaluation}

We used D1 cc, D3 cc, D5 cc, and D10 cc (minimum dose to volume of the most irradiated organ); and V5, V10, V15, $\mathrm{V} 20, \mathrm{~V} 25, \mathrm{~V} 30, \mathrm{~V} 35, \mathrm{~V} 40$, and V45 (minimum relative target volume in cc to dose of the most irradiated organ) to evaluate the OARs sparing for the duodenum, stomach, and small intestine. The $\mathrm{CI}$ of the plans was defined as the ratio between the volume of the $100 \%$ isodose line and the target volume. The CI was calculated with the following formula: 


$$
C I=\frac{V_{t, r e f}}{V_{t}} \cdot \frac{V_{t, r e f}}{V_{r e f}}
$$

where $V_{t, \text { ref }}$ is the volume of target area wrapped by $100 \%$ isodose line; $V_{t}$ is the target volume; and $V_{\text {ref }}$ is the volume of all areas wrapped by $100 \%$ isodose lines. The CI deviation ranged from 0 to 1 , with a higher CI value representing better conformality. The DVH and dose distribution diagrams of the patient with different dose patterns were acquired from the TPS. Additionally, the estimated treatment time for all the treatment planning techniques was also obtained from the HT TPS.

In a previous clinical study (18), patients received 20 fractions of radiotherapy, and the incidence of gastrointestinal toxicities side effects was relatively low, with no obvious grade 3-4 side effects. Therefore, we took the average tolerated dose of each OARs of patients receiving 20 fractions of radiotherapy as the standard dose. According to the equivalent bioradiotherapy formula for $B E D$ listed below, group B, group C and group D were converted into the average tolerated dose of each OARs corresponding to the number of fractions as the standard dose. The BED was calculated using the following formula:

$$
B E D=n d \times\left[1+\frac{X}{\alpha / \beta}\right]
$$

where $\mathrm{n}$ is the number of fractions, $\mathrm{d}$ is the dose per fraction, and $\alpha / \beta$ for tumors $=10$.nd as a whole; if $X=n d$, the $\mathrm{BED}$ formula can be converted to the following formula:

$$
B E D=X \times\left[1+\frac{X}{\frac{\alpha}{\beta} \times n}\right]
$$

where $\mathrm{n}$ is the number of fractions, $\mathrm{X}$ is the total irradiation dose, and $\alpha / \beta$ for tumors $=10$ (BED10).

In the hypofractionated radiotherapy mode, we estimated the patient's tolerance dose for OARs according to the number of fractions, especially for the tolerance dose for the gastrointestinal tract. If there was no statistically significant difference in the gastrointestinal tolerance dose between the hypofractionated radiotherapy mode with a certain number of fractions and the 20-fraction mode of radiation therapy, it would indicate the hypofractionated radiotherapy mode of treatment to be acceptable.

\section{Statistical methods}

The radiation dose variables of each OAR were expressed as mean \pm standard deviation for the normal or skewed distribution. The paired $t$-test statistical method was used to analyze the statistical differences in dosimetric parameters, treatment time, and CIs between group A and the other groups for dose assessment of OARs with dose escalation. Dose assessment of GI in hypofractionated mode was performed using independent samples $t$-test. A boxplot was used to show the statistical results. All statistical tests were conducted using SPSS 25.0 statistical software (IBM Corp.), and a 2 -sided $\mathrm{P}$ value $<0.05$ was deemed statistically significant.

\section{Results}

\section{Treatment volumes}

Based on the coverage of PTV, GTV, GTV1, GTV2, and the constraints for OARs, we generated the clinical HT plans of the 20-fraction radiation therapy mode for 17 patients who were originally treated with HT radiation therapy. Figure 1 shows the DVH and dose distribution diagram of a patient with different dose patterns. The average volumes of PTV, GTV, GTV1, GTV2, and GTV3 were respectively $147.77 \pm 65.21 \mathrm{cc}$ (range, 83.92-316.04 cc), $47.09 \pm 30.72 \mathrm{cc}$ (range, $14.95-131.38 \mathrm{cc}$ ), $29.23 \pm 22.59 \mathrm{cc}$ (range, $7.38-91.54 \mathrm{cc}$ ), $16.52 \pm 15.54 \mathrm{cc}$ (range, 3.22-59.54 cc), and $7.87 \pm 9.93 \mathrm{cc}$ (range, $0.55-36.17 \mathrm{cc}$ ). With PTV as the reference point for normalization, the ratios of GTV, GTV1, GTV2, and GTV3 to PTV were 31.87\%, 19.78\%, $11.18 \%$, and $5.33 \%$, respectively.

\section{Dose assessment of OARs for dose escalation}

As shown in Table 1 , there was no significant difference ( $\mathrm{P}$ values all $>0.05$ ) between group A and the other groups in D1 cc, D3 cc, D5 cc, and D10 cc of the duodenum, stomach, and intestine. The differences for V5, V10, V15, V20, V25, V30, V35, V40, and V45 between group A and the other groups are also provided in Table 1. More specifically, the V5 values of the duodenum, stomach, and intestine in group A were significantly different from those in the other groups; the V10 and V15 values of the duodenum were also significantly different between group A and group D, while the other values of group A were 


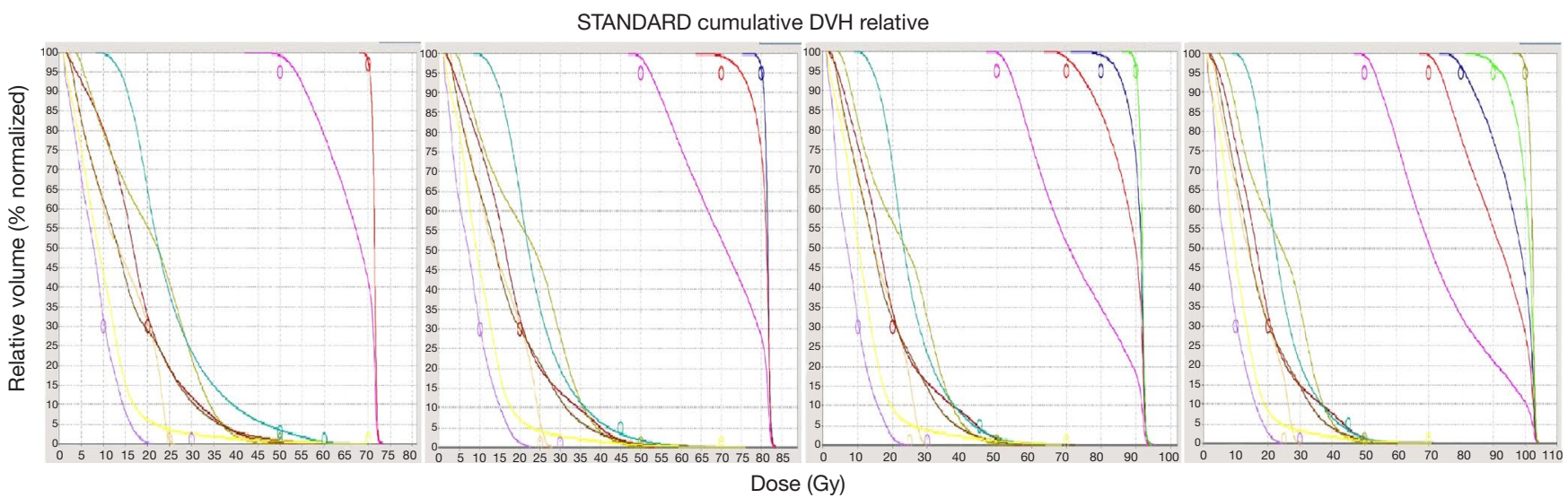

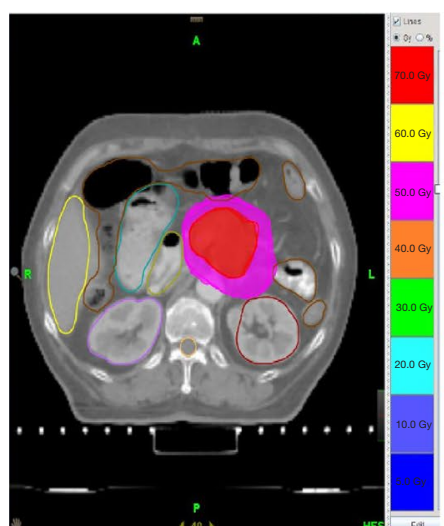

Group A

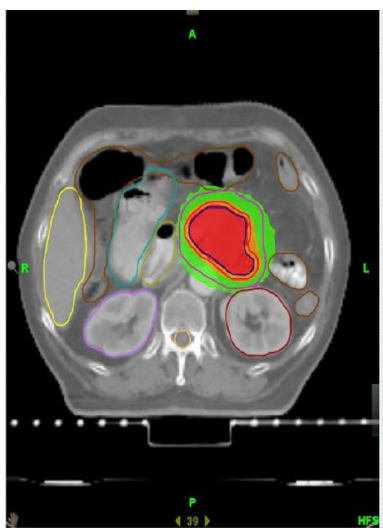

Group B

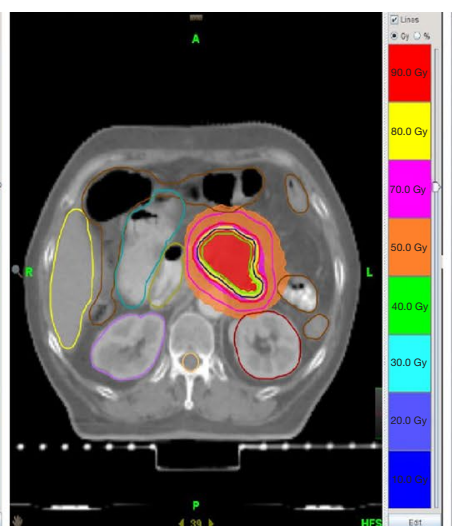

Group C

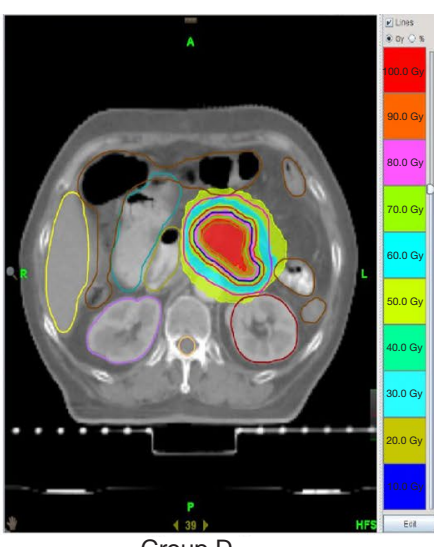

Group D

Figure $1 \mathrm{DVH}$ and dose distribution diagram of a representative patient with different dose patterns in groups A, B, C, and D. The top panel is DVH; the bottom panel is the dose distribution diagram. DVH, dose-volume histogram.

not statistically significant from those of the other groups. Compared with that of group A, the liver V30 of group D increased moderately by $0.342 \% \pm 1.908 \%$, which was not statistically significant (Figure 2). We noticed that the right and left kidney V20 and spinal cord Dmax of groups $\mathrm{B}, \mathrm{C}$, and D were all higher than those of group A (all P values $<0.05$ ) but all the doses of OARs were within the acceptable range. For example, the maximum dose of the spinal cord was $<32 \mathrm{~Gy}$, and the left and right kidney V20 values were $<35 \%$ (Figure 2). The therapy time of group D was increased by $21.147 \pm 10.256 \mathrm{sec}$. CI value of PTV was decreased by $0.056 \pm 0.060(\mathrm{P}<0.05)$ (Figure 3).

\section{Dose assessment of GI in the hypofractionated mode}

In group A, the irradiation dose was significantly different from the reference standard at the treatment of 10 fractions for the duodenum $(\mathrm{pD} 1=0.036)$, at the treatment of
12 fractions for the stomach ( $\mathrm{pD} 1=0.034)$, and at the treatment of 13 fractions for the intestine $(\mathrm{pD} 1=0.004$, $\mathrm{pD} 3=0.007, \mathrm{pD} 5=0.015, \mathrm{pD} 10=0.036$ ). In group $\mathrm{D}$, there was a significant difference at the treatment of 10 fractions for the duodenum ( $\mathrm{pD} 1=0.037)$, at the treatment of 11 fractions for the stomach $(\mathrm{pD} 1=0.012, \mathrm{pD} 3=0.032)$, and at the treatment of 14 fractions for the intestine $(\mathrm{pD} 1=0.029)$. Collectively, as highlighted in Table 2, the gastrointestinal dose-volume parameters started to become significantly different from the dose-limiting reference standard when the fraction number was reduced to less than 14 or 15 for group A or D, respectively (the full list of the dosimetric parameters is available in Table S1).

\section{Discussion}

Pancreatic cancer tissues are rich in fibrous tissues, low in immune cells and blood vessels. Due to this feature of the 
Table 1 Comparison of the dosimetric parameters for the duodenum, stomach, and small intestine between group A and other groups. A paired $t$-test was used to analyze the statistical difference

\begin{tabular}{|c|c|c|c|c|c|c|c|c|c|}
\hline Variable & \multicolumn{3}{|c|}{ Duodenum } & \multicolumn{3}{|c|}{ Stomach } & \multicolumn{3}{|c|}{ Intestine } \\
\hline D1 & 0.688 & 0.401 & 0.356 & 0.740 & 0.389 & 0.283 & 0.978 & 0.832 & 0.701 \\
\hline D3 & 0.135 & 0.535 & 0.749 & 0.811 & 0.496 & 0.231 & 0.723 & 0.566 & 0.976 \\
\hline D5 & 0.308 & 0.943 & 0.650 & 0.620 & 0.629 & 0.264 & 0.333 & 0.136 & 0.669 \\
\hline V5 & $0.022^{*}$ & 0.126 & $0.018^{*}$ & $0.014^{*}$ & 0.594 & $0.001^{*}$ & $0.029^{*}$ & $0.026^{\star}$ & $0.011^{*}$ \\
\hline V10 & 0.148 & 0.145 & $0.026^{\star}$ & 0.188 & 0.166 & 0.052 & 0.505 & 0.926 & 0.942 \\
\hline V15 & 0.278 & 0.063 & $0.035^{\star}$ & 0.666 & 0.411 & 0.259 & 0.374 & 0.249 & 0.257 \\
\hline V20 & 0.095 & 0.091 & 0.071 & 0.885 & 0.508 & 0.511 & 0.183 & 0.212 & 0.098 \\
\hline V35 & 0.914 & 0.077 & 0.057 & 0.387 & 0.849 & 0.283 & 0.095 & 0.060 & 0.371 \\
\hline V40 & 0.411 & 0.423 & 0.202 & 0.298 & 0.682 & 0.769 & 0.176 & 0.188 & 0.204 \\
\hline V45 & 0.091 & 0.116 & 0.369 & 0.195 & 0.249 & 0.486 & 0.586 & 0.635 & 0.665 \\
\hline
\end{tabular}

*, P value $<0.05$.

tumor microenvironment, conventional chemotherapy drugs, immune drugs, anti-angiogenic drugs, and conventional dose radiotherapy are often less effective for pancreatic cancer. Therefore, the treatment of pancreatic cancer requires novel, unconventional methods to improve the therapeutic outcome. As a special IMRT, HT allows $360^{\circ}$ rotational irradiation with 51 radiation fields combined with a binary aerodynamic multiblade collimator. The blade movement speed of HT is equivalent to $250 \mathrm{~cm} / \mathrm{s}$, 100 times higher than the speed of a traditional multiblade collimator (20). Dosimetric research for pancreatic cancer shows that HT has better conformal and focusing performance than ordinary IMRT technology (9). HT can adjust the dose in different target areas, and therefore allow the increase of the internal dose in the target area while keeping the peripheral dose to achieve the optimal effect of dose escalation. In the SIB mode, we applied high dose irradiation inside the target area and relatively low dose irradiation to the surrounding area, which not only restricted gastrointestinal toxicities, but also improved the local control of the tumor. Also, pathological studies and clinical observation revealed that different tumor areas may require different doses of radiation, which can be determined by the biological characteristics of solid tumor
$(21,22)$. Thus, dose escalation using HT in the SIB mode can also benefit the treatment of tumors in this regard.

In this study, the internal dose in the target area was further increased from the original 70 Gy (GTV), and the effect of dose escalation on corresponding OARs was evaluated at the dosimetric level. The dosimetric parameters are important indicators of gastrointestinal toxicities. Huang et al. illustrated that V20-V35 were predictive factors of gastrointestinal toxicity in patients receiving concurrent fractionated RT and gemcitabine (23). Verma et al. demonstrated that duodenal histopathologic damage, but not clinical symptoms, was correlated with duodenal mean dose, V35, V30, V25, V20, and mean/maximum PTV dose (24). Our results showed that the dose of the target area can be gradually increased to $100 \mathrm{~Gy}$ (group D) without causing a significant increase of the dose in the gastrointestinal tract compared with that in the control of group A (70 Gy). Specifically, there was no statistically significant difference (all $\mathrm{P}$ values $>0.05$ ) between group A and the other groups in D1 cc, D3 cc, D5 cc, D10cc, and V20-45 of the duodenum, stomach, and intestine. Therefore, the simultaneous boost of dosage to 100 Gy (group D) did not cause the risk of gastrointestinal tolerance dose to increase. Although the radiation dose of 

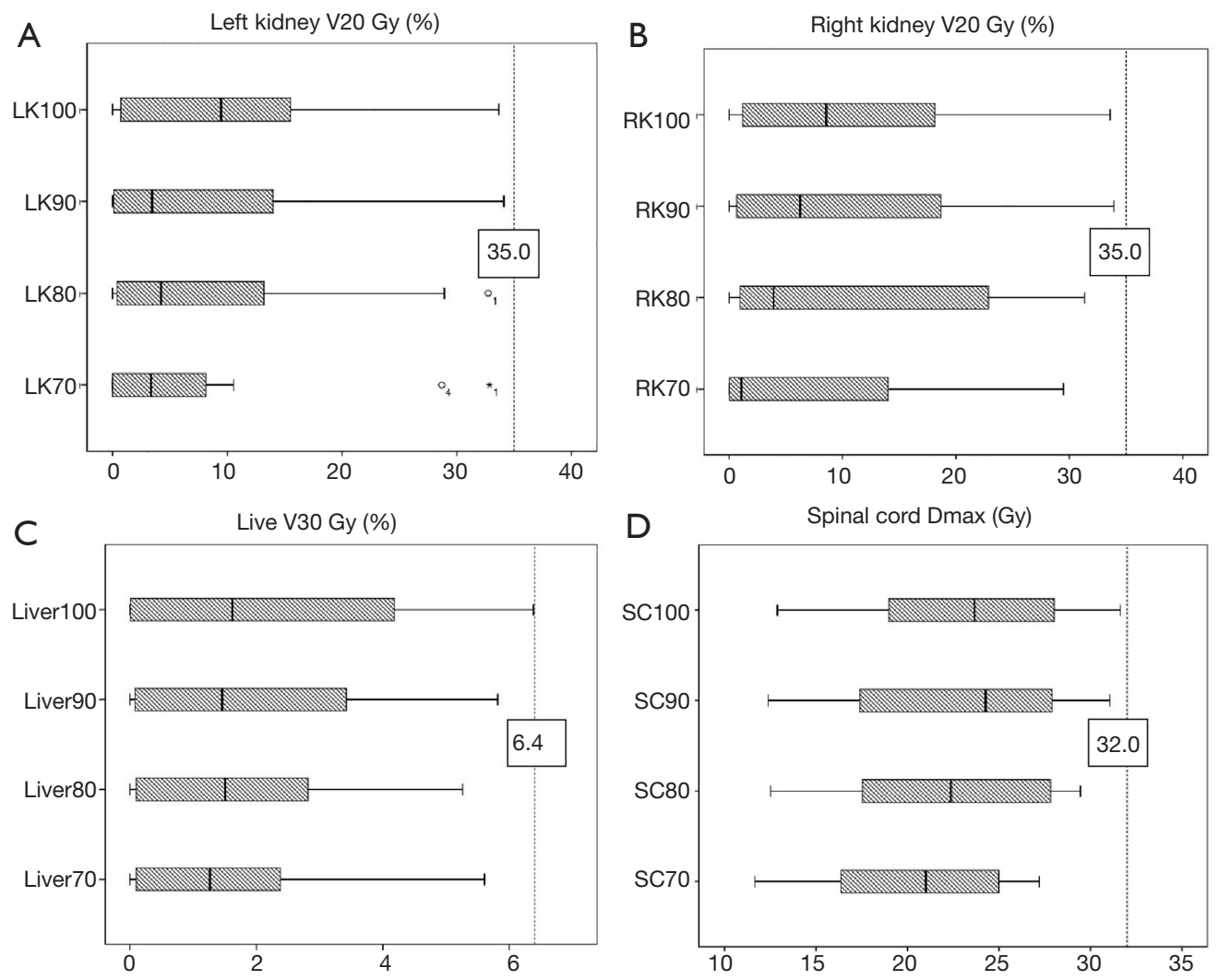

Figure 2 Average doses of different groups in the liver, kidney, and spinal cord. LK70, RK70, Liver70, and SC70 represent group A; LK80, RK80, Liver80, and SC80 represent group B; LK90, RK90, Liver90, and SC90 represent group C; and LK100, RK100, Liver100, and SC100 represent group D.
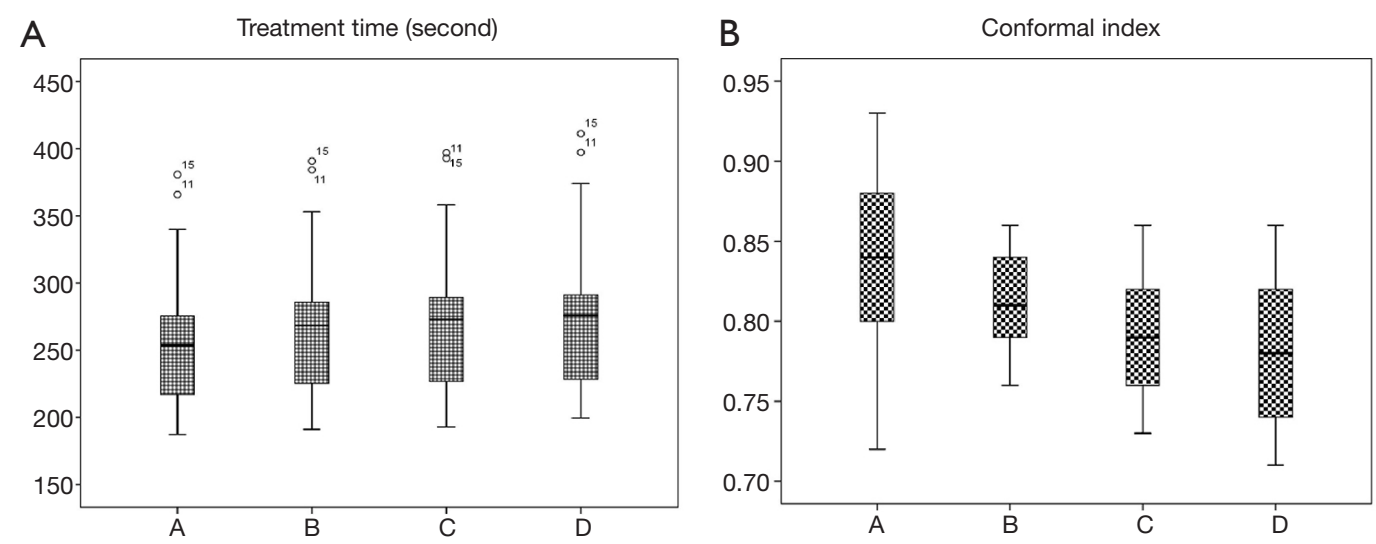

Figure 3 Average treatment time and target area conformal index (CI) of groups A, B, C, and D. 
Table 2 Comparison of the dosimetric parameters for the duodenum, stomach, and intestine in groups A and D vs. the reference standards with different numbers of fractions. A sample $t$-test was used to analyze the statistical difference

\begin{tabular}{|c|c|c|c|c|c|c|c|c|c|c|c|c|c|}
\hline \multicolumn{2}{|c|}{ Variable } & \multicolumn{4}{|c|}{ Duodenum $(\mathrm{N}=10)$} & \multicolumn{4}{|c|}{ Stomach $(\mathrm{N}=12)$} & \multicolumn{4}{|c|}{ Intestine ( $\mathrm{N}=14)$} \\
\hline$A$ & BED & $\frac{\mathrm{D} 1}{51.205}$ & $\frac{\text { D3 }}{44.346}$ & $\frac{\text { D5 }}{40.307}$ & $\frac{\mathrm{D} 10}{34.005}$ & $\frac{D 1}{51.230}$ & $\frac{\text { D3 }}{45.742}$ & $\frac{\text { D5 }}{42.979}$ & $\frac{\mathrm{D} 10}{37.927}$ & $\frac{D 1}{51.683}$ & $\frac{\text { D3 }}{46.391}$ & $\frac{\text { D5 }}{43.244}$ & $\begin{array}{c}\mathrm{D} 10 \\
38.785\end{array}$ \\
\hline & $\mathrm{P}$ & $0.036^{*}$ & 0.058 & 0.079 & 0.164 & 0.088 & 0.165 & 0.214 & 0.320 & 0.055 & 0.051 & 0.074 & 0.087 \\
\hline $\mathrm{D}$ & BED & 51.765 & 44.221 & 40.455 & 34.272 & 52.655 & 47.318 & 44.347 & 39.257 & 52.035 & 46.829 & 43.911 & 39.358 \\
\hline
\end{tabular}

*, $\mathrm{P}$ value $<0.05$. Only the fractions in which significant differences started to appear are presented here. The complete data set for all tested numbers of fractions can be seen in Table S1. Italic values indicate statistic difference.

the kidneys, liver, and spinal cord all increased, the increased value was still less than the dose limit. In particular, when the internal dose increased to $100 \mathrm{~Gy}$, the radiation dose of all OARs still complied with the dose limit: V30 for the liver was $<6.4 \%$, the maximum dose for the spinal cord was $<32 \mathrm{~Gy}$, and the V20 values for the kidney were $<35 \%$. The treatment time was increased by 21.15 seconds on average in group $\mathrm{D}$, in a range in which the prolapse was generally acceptable for patients. Regarding the CI of the plans, even though there was a significant difference between group A and group $\mathrm{D}$ ( $\mathrm{P}$ value $<0.01$ ), the conformity of group $\mathrm{D}$ was still high, with a CI of 0.78 .

BED10 in the high-dose hypofractionated radiation therapy mode needs to be higher than that in the conventional fractionated mode for improved outcomes. On the contrary, if BED10 is not significantly increased upon fraction number reduction, it indicates the effect on the prognosis of pancreatic cancer is limited. Using univariate analysis, Chang et al. found that BED >70 Gy could prolong overall survival in pancreatic patients; using the failure mode analysis, they further showed that BED $>70$ Gy was an independent prognostic factor for local, regional, and distant failure-free survival (25). Krishnan et al. retrospectively analyzed the prognosis of 200 patients with LAPC who received radiotherapy, including 47 patients with BED >70 Gy (15). The results indicated that the patients receiving BED $>70$ Gy had a higher 2-year overall survival rate (36\% versus $19 \%$ ). Furthermore, their multivariate analysis revealed that BED was the only independent prognostic factor for survival. Meanwhile, Lin et al. retrospectively investigated the differences in efficacy between the hypofractionated pattern and the conventional pattern: 20 patients in the hypofractionated group received $35-45$ Gy, in a 7-9 Gy/fraction, which, when converted to
BED10, was 59.5-85.5 Gy; 21 patients in the conventional group received 45-50.4 Gy, in a 1.8-2 Gy/fraction, which, when converted to BED10, was 54-60 Gy (26). Their results indicated that the treatment of pancreatic cancer with a hypofractionated pattern could achieve a higher local disease-free survival than conventional treatment $(\mathrm{P}=0.004)$. The median survival time was 20 months for the hypofractionated pattern, and 13 months for the conventional pattern. Overall, the previous studies have suggested that the hypofractionated mode could be superior to the conventional mode. However, to the best of our knowledge, there have been no randomized, controlled comparison studies conducted in this regard. In addition, no such dosimetric study has been performed to investigate HT techniques for pancreatic cancer.

Based on the determination of the internal dose at the dosimetric level, we sought to identify the optimal fraction number of HT radiotherapy techniques for the treatment of LAPC. Without further raising the gastrointestinal dose, decreasing the treatment fraction number can result in an increase of BED10, which can be beneficial to improving the therapeutic effect and reducing the economic burden on patients and the pressure on medical workers. Based on our study, under the condition of PTV 50 Gy and GTV 70 Gy, when the fraction number is 15 , we can gradually maximize the target irradiation dose up to 100 Gy without increasing the surrounding gastrointestinal dose.

In recent years, hypofractionated radiotherapy has attracted increased attention in the radiotherapy field for treating various cancers, including lung cancer, liver cancer, prostate cancer, etc. (27-29). As a result, the previous cancer treatment mode has gradually switched to a more rapid and effective hypofractionated radiotherapy mode. Due to the extensive involvement of the pancreas with other organs, it 
is more difficult to precisely increase the radiation dose in the target area of the pancreatic tumor than it is in other tumors.

Admittedly, there were some limitations to this study. The effects of positioning error and respiratory movement could have affected the accuracy of irradiation in the process of hypofractionated radiotherapy for the dosimetric study. Additionally, more verification of using HT techniques for LAPC in the clinical research setting is still necessary to determine how to further improve the internal dose in the target area and reduce the number of fractions. Despite these limitations, the findings of this dosimetric study demonstrate that the use of HT techniques for LAPC is a promising and powerful approach to increase the radiation dose for improving clinical treatment.

\section{Conclusions}

In this study, we demonstrated that in using HT techniques for LACP, dose constraints are achievable for OARs, including the kidney, liver, spinal cord, and especially the gastrointestinal tract. Our findings give credence to the feasibility of dose escalation for LAPC, and provide evidence that the target irradiation dose can be gradually increased to 100 Gy without increasing the surrounding gastrointestinal dose. Our data provide a hypofractionated radiotherapy model for other clinicians seeking dose escalation for pancreatic cancer, which can be set to 100 Gy in 15 fractions without increasing the surrounding gastrointestinal dose.

\section{Acknowledgments}

Funding: None.

\section{Footnote}

Reporting Checklist: The authors have completed the MDAR reporting checklist. Available at http://dx.doi.org/10.21037/ jgo-21-160

Data Sharing Statement: Available at http://dx.doi. org/10.21037/jgo-21-160

Conflicts of Interest: All authors have completed the ICMJE uniform disclosure form (available at http://dx.doi. org/10.21037/jgo-21-160). The authors have no conflicts of interest to declare.
Ethical Statement: The authors are accountable for all aspects of the work in ensuring that questions related to the accuracy or integrity of any part of the work are appropriately investigated and resolved. Verbal informed consent was obtained from all patients. This study is dosimetric in nature and thus did not require an ethical board approval, as it did not involve any animal or human experiments or interventions. The study was conducted in accordance with the Declaration of Helsinki (as revised in 2013).

Open Access Statement: This is an Open Access article distributed in accordance with the Creative Commons Attribution-NonCommercial-NoDerivs 4.0 International License (CC BY-NC-ND 4.0), which permits the noncommercial replication and distribution of the article with the strict proviso that no changes or edits are made and the original work is properly cited (including links to both the formal publication through the relevant DOI and the license). See: https://creativecommons.org/licenses/by-nc-nd/4.0/.

\section{References}

1. Siegel RL, Miller KD, Jemal A. Cancer statistics, 2020. CA Cancer J Clin 2020;70:7-30.

2. Ren G, Xia T, Wang Y. IMRT with capecitabine in advanced pancreatic cancer. In regard to Passoni et al. Int J Radiat Oncol Biol Phys 2014;89:431.

3. Reyngold M, Parikh P, Crane CH. Ablative radiation therapy for locally advanced pancreatic cancer: techniques and results. Radiat Oncol 2019;14:95.

4. Lewis S, Sastri SC, Arya S, et al. Dose escalated concurrent chemo-radiation in borderline resectable and locally advanced pancreatic cancers with tomotherapy based intensity modulated radiotherapy: a phase II study. J Gastrointest Oncol 2019;10:474-82.

5. Chauffert B, Mornex F, Bonnetain F, et al. Phase III trial comparing intensive induction chemoradiotherapy (60 Gy, infusional 5-FU and intermittent cisplatin) followed by maintenance gemcitabine with gemcitabine alone for locally advanced unresectable pancreatic cancer. Definitive results of the 2000-01 FFCD/SFRO study. Ann Oncol 2008;19:1592-9.

6. Loehrer PJ Sr, Feng Y, Cardenes H, et al. Gemcitabine alone versus gemcitabine plus radiotherapy in patients with locally advanced pancreatic cancer: an Eastern Cooperative Oncology Group trial. J Clin Oncol 2011;29:4105-12.

7. Hammel P, Huguet F, van Laethem JL, et al. Effect 
of Chemoradiotherapy vs Chemotherapy on Survival in Patients With Locally Advanced Pancreatic Cancer Controlled After 4 Months of Gemcitabine With or Without Erlotinib: The LAP07 Randomized Clinical Trial. JAMA 2016;315:1844-53.

8. Kumar R, Wild AT, Ziegler MA, et al. Stereotactic body radiation therapy planning with duodenal sparing using volumetric-modulated arc therapy vs intensity-modulated radiation therapy in locally advanced pancreatic cancer: A dosimetric analysis. Med Dosim 2013;38:243-50.

9. Taylor R, Opfermann K, Jones BD, et al. Comparison of radiation treatment delivery for pancreatic cancer: Linac intensity-modulated radiotherapy versus helical tomotherapy. J Med Imaging Radiat Oncol 2012;56:332-7.

10. Boldrini L, Cusumano D, Cellini F, et al. Online adaptive magnetic resonance guided radiotherapy for pancreatic cancer: state of the art, pearls and pitfalls. Radiat Oncol 2019;14:71.

11. Takahashi S, Anada M, Kinoshita T, et al. Respiratory motion of lymph node stations in pancreatic cancer: Analyses using contrast-enhanced four-dimensional computed tomography. Radiother Oncol 2018;128:569-74.

12. Teboh RF, Srinivasan S, Ng SP, et al. Setup Management for Stereotactic Body Radiation Therapy of Patients With Pancreatic Cancer Treated via the Breath-Hold Technique. Pract Radiat Oncol 2020;10:e280-e289.

13. Wang J, Xia T, Wang Y, et al. Long-term results of gamma ray-based stereotactic body radiotherapy in treatment of medically unfit or inoperable non-metastatic pancreatic adenocarcinoma[Abstract 3587]. Int J Radiat Oncol Biol Phys 2012;84:S815-6.

14. Nabavizadeh N, Simeonova AO, Waller JG, et al. Volumetric-modulated arc radiotherapy for pancreatic malignancies: dosimetric comparison with sliding-window intensity-modulated radiotherapy and 3-dimensional conformal radiotherapy. Med Dosim 2014;39:256-60.

15. Krishnan S, Chadha AS, Suh Y, et al. Focal Radiation Therapy Dose Escalation Improves Overall Survival in Locally Advanced Pancreatic Cancer Patients Receiving Induction Chemotherapy and Consolidative Chemoradiation. Int J Radiat Oncol Biol Phys 2016;94:755-65.

16. Herman JM, Chang DT, Goodman KA, et al. Phase 2 multi-institutional trial evaluating gemcitabine and stereotactic body radiotherapy for patients with locally advanced unresectable pancreatic adenocarcinoma. Cancer 2015;121:1128-37.

17. Bertholet J, Hunt A, Dunlop A, et al. Comparison of the dose escalation potential for two hypofractionated radiotherapy regimens for locally advanced pancreatic cancer. Clin Transl Radiat Oncol 2019;16:21-7.

18. Xia T, Chang D, Wang Y, et al. Dose escalation to target volumes of helical tomotherapy for pancreatic cancer in the phase I-II clinical trial [abstract 2262]. Int J Radiat Oncol Biol Phys 2013;87:S303.

19. Ren G, Xia TY, Di YP, et al. Hypofractionated and simultaneous integrated boost radiation therapy for locally advanced pancreatic cancer with helical tomotherapy. Int J Radiat Oncol Biol Phys 2015;93:E149-E150.

20. Yartsev S, Kron T, Van Dyk J. Tomotherapy as a tool in image-guided radiation therapy (IGRT): theoretical and technological aspects. Biomed Imaging Interv J 2007;3:e16.

21. Li F, Zhou K, Gao L, et al. Radiation induces the generation of cancer stem cells: A novel mechanism for cancer radioresistance. Oncol Lett 2016;12:3059-65.

22. Ren G, Zhu F, Xia T, et al. Dosimetric study on dose escalation in internal target of pancreatic cancer with helical tomotherapy. Int J Radiat Oncol Biol Phys 2014;90:S357.

23. Huang J, Robertson JM, Ye H, et al. Dose-volume analysis of predictors for gastrointestinal toxicity after concurrent full-dose gemcitabine and radiotherapy for locally advanced pancreatic adenocarcinoma. Int J Radiat Oncol Biol Phys 2012;83:1120-5.

24. Verma V, Lazenby AJ, Zheng D, et al. Dosimetric parameters correlate with duodenal histopathologic damage after stereotactic body radiotherapy for pancreatic cancer: Secondary analysis of a prospective clinical trial. Radiother Oncol 2017;122:464-9.

25. Chang JS, Wang ML, Koom WS, et al. High-dose helical tomotherapy with concurrent full-dose chemotherapy for locally advanced pancreatic cancer. Int J Radiat Oncol Biol Phys 2012;83:1448-54.

26. Lin JC, Jen YM, Li MH, et al. Comparing outcomes of stereotactic body radiotherapy with intensitymodulated radiotherapy for patients with locally advanced unresectable pancreatic cancer. Eur J Gastroenterol Hepatol 2015;27:259-64.

27. Chang JY, Senan S, Paul MA, et al. Stereotactic ablative radiotherapy versus lobectomy for operable stage I nonsmall-cell lung cancer: a pooled analysis of two randomised trials. Lancet Oncol 2015;16:630-7.

28. Wahl DR, Stenmark MH, Tao Y, et al. Outcomes after stereotactic body radiotherapy or radiofrequency ablation for hepatocellular carcinoma. J Clin Oncol 2016;34:452-9. 
29. Wilkins A, Mossop H, Syndikus I, et al. Hypofractionated radiotherapy versus conventionally fractionated radiotherapy for patients with intermediate-risk localised prostate cancer: 2-year patient-reported outcomes of the

Cite this article as: Teng F, Meng L, Zhu F, Ren G. Dosimetric feasibility on hypofractionated intensity-modulated radiotherapy and simultaneous integrated boost for locally advanced unresectable pancreatic cancer with helical tomotherapy. J Gastrointest Oncol 2021;12(2):496-506. doi: 10.21037/jgo-21-160 randomised, non-inferiority, phase $3 \mathrm{CHHiP}$ trial. Lancet Oncol 2015;16:1605-16.

(English Language Editor: J. Gray) 
Supplementary

Table S1 Comparison of the dosimetric parameters for the duodenum, stomach, and intestine between group A and group D with different numbers of fractions. A sample $t$-test was used to analyze the statistical difference

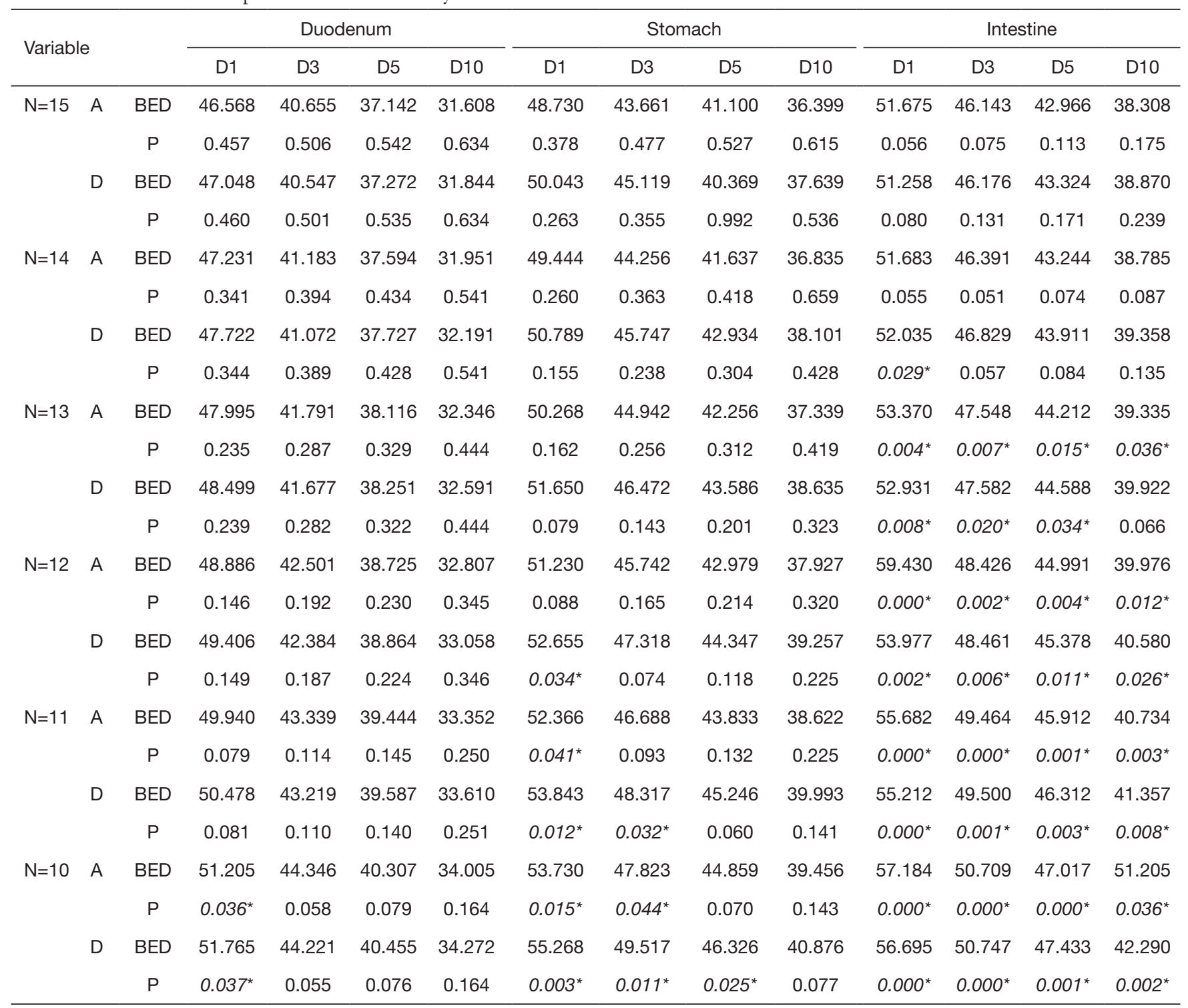

${ }^{*}$, $\mathrm{P}$ value $<0.05$. Italic values indicate statistic difference. 